\title{
De Uribe a Santos: Cambios y continuidades DE LA POLÍTICA COLOMBIANA EN 2011
}

\author{
From Uribe to Santos: Changes and continuities \\ in 2011 Colombian politics
}

\section{LAURA WILLS-OTERO* \\ Universidad de los Andes, Bogotá \\ CHRISTIAN IVÁN BENITO}

Universidad de los Andes, Bogotá

\begin{abstract}
RESUMEN
El siguiente artículo tiene como objetivo reseñar algunos de los hechos, procesos y decisiones de carácter público que definieron la política nacional colombiana durante 2011. A lo largo del año, el gobierno de Juan Manuel Santos tomó decisiones que reflejaron cambios con respecto a las políticas de su antecesor, Álvaro Uribe. En este documento se hace énfasis en ello. Dada la importancia que adquirieron las elecciones presidenciales y legislativas de 2010, acá se incluye un balance de las mismas. Las siguientes secciones resumen temas sobresalientes de la realidad nacional; resultados electorales de comicios nacionales y locales; cambios institucionales sustantivos; y aspectos que caracterizan a las ramas del poder público.
\end{abstract}

Palabras clave: Juan Manuel Santos, electores, Poder Ejecutivo.

\begin{abstract}
This article highlights some of the key events, processes and public policy decisions that have defined Colombian politics throughout 2011. Juan Manuel Santos's government has made decisions that have distanced his administration from his predecessor Alvaro Uribe's policies. This document focuses on these differences. It offers an overview of the 2010 presidential and legislative elections given their importance in defining national politics. The following sections summarize highly salient national issues, nationwide and local electoral results, substantial institutional changes, as well as other relevant aspects that characterize Colombian branches of government.
\end{abstract}

Key words: Juan Manuel Santos's, electoral, Executive Power. 


\section{TEMAS SOBRESALIENTES DE LA REALIDAD NACIONAL}

\subsection{Coyuntura social}

Uno de los temas de la coyuntura social que sobresalieron en 2011 fue la alta movilización por parte de los ciudadanos y de sectores de la sociedad civil que de manera organizada manifestaron su posición frente a políticas promovidas por el Gobierno Nacional. En primer lugar, uno de los anuncios que hizo el gobierno al iniciar el año fue la promulgación de un decreto en el cual se modificaría la regulación existente en el sector de transportadores de carga por carretera (i.e. la llamada tabla de fletes). La reforma estaba dirigida a eliminar la intervención estatal en el establecimiento de los precios que se les paga a los camioneros por cada trayecto y a transitar a un régimen de libre oferta y demanda. La noticia generó gran resistencia en los transportadores quienes denunciaban que la medida llevaría a la pérdida de empleo de una gran cantidad de personal. Como reacción al decreto, los afectados se organizaron en el principal gremio del sector, la Asociación Colombiana de Camioneros (ACC), y a través de un paro nacional que se prolongó por dos semanas, así como de bloqueos en las principales vías del país, lograron llegar a un acuerdo con el gobierno en el que éste se comprometió a retirar los decretos reformatorios y a concertar con las partes afectadas un nuevo sistema regulatorio basado en los costos operativos y la intervención del Estado cuando existan fallas de mercado que así lo ameriten (León, 2011; Portafolio, 2011a; Semana, 2011d).

Un segundo tema que también dio pie para que la ciudadanía se movilizara fue otra propuesta de iniciativa gubernamental dirigida a realizar una reforma sustantiva a la ley nacional de educación (Ley 30 de 1992). Se planteaba la necesidad de involucrar al sector privado en la financiación de la educación, y crear nuevas instituciones privadas con ánimo de lucro para generar más cupos y aumentar con ello la cobertura. Si bien la iniciativa se convirtió en un proyecto de ley que fue radicado en el último trimestre del año (octubre), la presentación de la idea por parte de la ministra de Educación, María Fernanda Campo, se había realizado desde marzo. La reacción del sector educativo a la iniciativa fue inmediata. Desde abril y durante varios meses, estudiantes universitarios y profesores de instituciones públicas fundamentalmente pero también privadas salieron a protestar a lo largo y ancho del país (La Silla Vacía, 2011). En su opinión, la reforma promovería la privatización. Esto generaría un precio muy alto para la sociedad, principalmente para quienes no tienen capacidad financiera para acceder a cupos pagos. Además, el afán por generar más puestos no iba de la mano de una propuesta dirigida a aumentar la calidad de la educación. La inconformidad por parte del sector educativo resultó en un paro nacional que inició a principios de octubre y se extendió hasta mediados de noviembre. A éste se sumaron las universidades públicas del país las cuales cancelaron sus clases. El paro se levantó una vez que el gobierno decidió retirar del Congreso de la República el proyecto de ley. Con ello, la voluntad de la sociedad se volvió a imponer sobre la de las autoridades políticas (CVNE, 2011; El País, 2011).

Dejando a un lado las manifestaciones sociales, uno de los problemas que más costos le causó a la población colombiana en 2011 fue el referido a "la ola invernal" iniciado en 2010. La catástrofe natural causada por el fenómeno de "La Niña" sacudió al país casi 
en su totalidad con la ocurrencia de incontables inundaciones, desbordamiento de ríos, derrumbes en carreteras, miles de muertos y cerca de tres millones de damnificados (Semana, 2011a). Diferentes sectores de la economía nacional - ej: infraestructura, industria, agricultura y ganadería- se vieron fuertemente afectados por la prolongación de las lluvias (Vargas, 2011). Ante este panorama, el gobierno central destinó gran cantidad de recursos a los municipios afectados (alrededor de 4 billones de pesos), lo cual significó el recorte del gasto social en algunos rubros.

\subsection{Coyuntura económica}

A pesar de la crisis financiera que vivieron algunas de las principales economías del mundo durante el 2011, y del esperado efecto dominó que la caída de las bolsas europeas y asiáticas produciría en las economías emergentes, la economía colombiana logró ajustarse y contrarrestar el impacto negativo. Muestra de ello, es que los principales indicadores macroeconómicos del país presentaron tendencias favorables a la vez que se registraron avances significativos en varias de las metas que se fijó el gobierno de Juan Manuel Santos en temas como la reducción del desempleo, la estabilización de la inflación y el incremento del producto interno bruto (PIB) per cápita. Este último pasó de US\$ 6.225 en 2010 a US\$ 6.700 en 2011. ${ }^{1}$ Por su parte, aunque la inflación presentó un ligero incremento, se mantuvo en un dígito y estuvo en el rango del $2 \%$ al $4 \%$ que el banco central estableció el año anterior (2010). Tal nivel mantendría la confianza en la economía. El desempleo bajó del 11,8\% en 2010 a 9,7\% en 2011. Con ello se cumplió el objetivo que se planteó el Ministerio de Hacienda de reducir esta cifra a un solo dígito (entre el 8 y el 9\%) a través de la creación de 600.000 nuevos puestos de trabajo anuales. ${ }^{2}$ No obstante este éxito, algunas centrales obreras del país plantean que el desempleo está por encima de los dos dígitos, argumentando que el gobierno no tiene en cuenta el sector informal de la economía nacional (Revista Dinero, 2011). El Cuadro 1 presenta información sobre los principales indicadores macroeconómicos para el período 2007-2011.

Cuadro 1: Principales indicadores macroeconómicos en Colombia (2007-2011)

\begin{tabular}{lrrrcc}
\hline & 2007 & 2008 & 2009 & 2010 & 2011 \\
\hline PIB per cápita* & 4.676 & 5.436 & 5.166 & 6.225 & 6.700 \\
Inflación (\% anual) & 5,5 & 7,0 & 4,2 & 3,17 & 3,73 \\
Desempleo total (\%) & 10,3 & 11,4 & 12,0 & 11,8 & $9,7^{3}$ \\
Déficit** & $-1,8$ & $-6,8$ & $-4,0$ & $-3,2$ & $-2,2$ \\
\hline
\end{tabular}

* Datos en US\$ a precios actuales.

** Déficit medido como puntos porcentuales del PIB. El valor negativo representa la deuda del gobierno nacional.

Fuentes: Banco Mundial (2008-2012) y DANE. Elaboración propia.

1 Es preciso señalar que desde 2007 este indicador presenta una tendencia incremental que en teoría es favorable en el bienestar de los colombianos.

2 La meta está establecida para ser cumplida hasta el 2014. 
En cuanto a las finanzas públicas, el país se mantuvo en una situación de déficit a pesar de que desde 2008 el indicador empezó a presentar algunas mejoras. En 2011 el déficit fiscal fue de $\$ 13.354$ miles de millones de pesos (2\% del PIB) frente a $\$ 17.713$ miles de millones de pesos en 2010 (3,2\% del PIB). Una de las metas del actual gobierno apunta a que en el 2012 el déficit no supere dos puntos porcentuales del PIB. Teniendo en cuenta el estado deficitario de las finanzas públicas, durante el 2011 el Ejecutivo diseñó una medida de ajuste estructural conocida como "la regla fiscal". Con esta, se espera la reducción progresiva de la deuda (Banco de la República, 2010). De igual manera, en marzo de 2012 el presidente Santos, por intermedio de su ministro de Hacienda Juan Carlos Echeverri, presentará al Congreso de la República una reforma tributaria que tratará de contribuir al saneamiento de las cuentas nacionales. El objetivo de la reforma es obtener recursos para impulsar la economía nacional sin el incremento en los impuestos.

\subsection{Coyuntura subregional e internacional}

La llegada de Juan Manuel Santos a la presidencia en agosto de 2010 significó para el país un giro en la dinámica de las relaciones con los países vecinos, especialmente con Venezuela y Ecuador. Desde su posesión, el mandatario decidió restablecer los vínculos que durante el gobierno de su antecesor, Álvaro Uribe, se vieron afectados fundamentalmente por tensiones relacionadas con el conflicto armado interno. ${ }^{3}$ Cuatro días después de la inauguración de su mandato, Santos se reunió con Chávez en Santa Marta (Colombia) y entre los dos decidieron restablecer las relaciones comerciales y diplomáticas (Semana, 2010), que habían sido rotas desde 2009 y 2010 respectivamente (El País, 2010). En marzo de 2011, los cancilleres de ambos países se reunieron en Caracas y firmaron trece acuerdos comerciales, con lo que se empezó a concretar la nueva etapa de cooperación entre los dos países (Lares, 2011). A lo largo del año (2011), diferentes encuentros entre los mandatarios o sus cancilleres y declaraciones por parte de uno $\mathrm{u}$ otro, evidenciaron un esfuerzo por mantener las buenas relaciones, y contrarrestar la opinión que a través de diferentes medios el ex presidente Uribe expresó sobre su desacuerdo en entablar vínculos con el gobierno chavista (El Nuevo Herald, 2011). Por su parte, las relaciones bilaterales con Ecuador también se encaminaron a fortalecer la integración (Portafolio, 2011b).

Otra demostración por parte del gobierno de Colombia de su propósito de fortalecer las relaciones regionales fue la postulación de la ex canciller María Emma Mejía para ocupar la secretaría general de Unasur. Su nombramiento a principios del año fue resaltado como una oportunidad para que Colombia se constituya en un país que lidere procesos de integración regional (Semana, 2011c). Trascendiendo el escenario latinoamericano, a partir de marzo de 2011 Colombia entró a presidir el Consejo de Seguridad de la

\footnotetext{
En más de una ocasión, Uribe denunció que Chávez protegía guerrilleros de las FARC en las fronteras de los dos países. Antes de finalizar su gobierno, Uribe interpuso denuncias contra Chávez ante la Corte Penal Internacional por violación de derechos humanos. Por su parte, Correa en Ecuador denunció violación a la soberanía de su país por parte del Estado colombiano cuando en 2008 las Fuerzas Militares colombianas abatieron a Raúl Reyes de las FARC en territorio ecuatoriano.
} 
Organización de las Naciones Unidas (ONU). Este hecho se constituyó en otra oportunidad para que el país muestre liderazgo en la comunidad internacional (El Tiempo, 2011a). Otro acontecimiento de la coyuntura internacional fue la firma del Tratado de Libre Comercio (TLC) entre Colombia y Estados Unidos la cual se logró después de cuatro años de espera (El Espectador, 2011b). Esto, sumado a la ratificación de otro TLC con la Unión Europea planteó más oportunidades de inserción internacional y crecimiento, siempre y cuando el país logre superar diversos obstáculos en el nivel interno, tales como la reconstrucción de carreteras tras su afectación por la ola invernal (Tickner, 2011).

\subsection{Coyuntura política}

Además de las elecciones locales que se realizaron en octubre (ver sección 4), los eventos más importantes de la coyuntura política en 2011 estuvieron relacionados con el conflicto armado interno por un lado, y con el destape de diversos escándalos de corrupción por el otro.

En cuanto al primer tema, el conflicto continuó pese a una posición más reconciliadora del gobierno de Juan Manuel Santos respecto a la de su antecesor. En diversos escenarios, el Presidente sugirió la posibilidad de entablar diálogos con la guerrilla -FARC y ELN-, siempre y cuando ésta diera muestras fehacientes de su voluntad de negociación (por ejemplo, con la liberación de secuestrados). Por su parte, desde la campaña electoral y posteriormente cuando Santos fue electo, las FARC declararon públicamente su disponibilidad de entablar conversaciones con el nuevo presidente (El Mundo, 2010). Claramente ambas posiciones -la del presidente y la de la guerrilla- marcaron una diferencia fundamental con respecto al carácter confrontacional que definió la política de Uribe a lo largo de sus ocho años de gobierno.

A pesar de la muestra de voluntad política de las dos partes, acontecimientos sucedidos a lo largo del año alejaron la posibilidad de la concreción de una salida negociada del conflicto. El grupo guerrillero arremetió contra el ejército y la policía en múltiples oportunidades en diferentes zonas del país (El Nuevo Siglo, 2011; El Tiempo, 2011c). A esto se sumó el secuestro en octubre de la niña Nhora Valentina Muñoz, hija del alcalde del municipio de Fortul en Arauca, y el cruel asesinato en noviembre de cuatro miembros de la fuerza pública que habían acumulado hasta catorce años en cautiverio (Semana, 2011b). Analistas plantearon que las arremetidas del grupo guerrillero no fueron más que retaliaciones por operaciones y capturas que las Fuerzas Militares realizaron en el 2011, así como por la aprobación en el Congreso de la República de políticas que amenazan su existencia (i.e. la ley de víctimas. Ver sección 3). Por su parte, el gobierno planteó que los diferentes hechos de violencia fueron un reflejo de la debilidad del grupo guerrillero y de su necesidad de llamar la atención sobre su existencia.

Frente a los diferentes ataques, el gobierno reaccionó fortaleciendo el pie de fuerza en algunas regiones (Arauca y Cauca), y realizando diferentes operaciones militares en las que se buscó dar de baja a guerrilleros y otros actores ilegales. El resultado de una de tales operaciones -la "Operación Odiseo" realizada en noviembre en el departamento de Cauca- fue el abatimiento del máximo cabecilla del grupo Alfonso Cano. Esta muerte se 
sumó a la de quien fuera el máximo líder del grupo, Manuel Marulanda, alias Tirofijo, en 2008 y a las de Raúl Reyes (2008) y el Mono Jojoy, quien fue dado de baja en la "Operación Sodoma" en septiembre de 2010, a menos de dos meses de inaugurado el gobierno de Santos. Tras la muerte de Cano, el secretariado del grupo eligió a Rodrigo Londoño Echeverri alias Timoleón Jiménez, o "Timochenko" como su reemplazo. En su primer mensaje al Presidente de la República, calificó a Santos como "amenazante y brutal" (El Tiempo, 2011b). Sin embargo, en posteriores comunicados volvió a plantear la posibilidad del diálogo con el gobierno a lo que el último respondió como imposible bajo las condiciones generadas por el grupo a lo largo de los últimos meses (León, 2012).

Además del conflicto con los grupos guerrilleros, durante el 2011 las llamadas "bandas criminales emergentes" o BACRIM sembraron el miedo en muchas regiones del país donde cometieron asesinatos, secuestros, desapariciones forzadas y extorsiones. ${ }^{4} \mathrm{Se}$ calcula que estas organizaciones armadas suman cerca de 10.000 hombres y que muchos de ellos provienen de los grupos paramilitares que se desmovilizaron durante el gobierno de Álvaro Uribe. Estudios han revelado que estos grupos tienen fuertes vínculos con actores vinculados con el negocio del narcotráfico (Arias Ortiz, 2012). Con sus actos y su presencia fortalecida en más de 200 municipios del país, las BACRIM se constituyen en un desafío adicional, en materia de orden público, para los representantes del Estado colombiano.

La otra cara de la coyuntura política se refiere al destape de diversos escándalos de corrupción en los que tanto funcionarios públicos como contratistas con el Estado, estuvieron involucrados. Los escándalos llevaron a la apertura de varias investigaciones penales por parte de la Fiscal General de la Nación, Viviane Morales, y a la liquidación de instituciones foco de corrupción (ver sección 2). A continuación se mencionan solo algunos de los escándalos que ocuparon más espacio en los medios durante el 2011. El primero de ellos se relaciona con el programa del gobierno de Uribe "Agro Ingreso Seguro", promovido por el ex ministro de Agricultura, Andrés Felipe Arias. El objetivo del programa era otorgar subsidios a agricultores y promover con ello el desarrollo rural. El escándalo que llevó a que tanto Arias como varios funcionarios del ministerio fueran detenidos, estalló cuando los medios revelaron que los beneficiarios del programa habían sido grandes propietarios de tierras y no los pequeños campesinos para quienes se habían definido los subsidios. En segundo lugar, en junio se destapó el que se llamó el "carrusel de la contratación", el cual se relacionó con la denuncia de múltiples irregularidades en la adjudicación de millonarios contratos en los que se vieron involucrados políticos y empresarios. El alcalde de Bogotá, Samuel Moreno Rojas; su hermano el senador Iván Moreno Rojas; los empresarios del conocido "Grupo Nule"; y otros funcionarios públicos fueron destituidos de sus cargos. Algunos de ellos ya fueron condenados a pagar varios años de cárcel. Otros todavía esperan la decisión judicial. Un destape más se dio en el sector de la salud. Se descubrió que recursos públicos del sector, se destinaron a

4 Algunas de ellas son conocidas como “Los Rastrojos", “Los Urabeños”, “Los Paisas”, “Las Águilas Negras” y "El Ejército Anticomunista de Colombia". 
particulares y no a los destinatarios naturales. Como en los otros casos, en este también se abrieron procesos contra varios implicados.

\section{CAMBIOS INSTITUCIONALES Y CAMBIOS SUBSTANCIALES EN POLÍTICAS PÚBLICAS}

En esta sección describimos algunos de los cambios institucionales más importantes, así como leyes que marcaron hitos en la política nacional en 2011. Sobre el primer aspecto, a inicios del año el Congreso de la República le otorgó a Juan Manuel Santos facultades extraordinarias para reestructurar el Estado. A través de decretos, el Presidente realizó los siguientes cambios: en primer lugar, revivió tres ministerios (Justicia, Trabajo y Salud) que Álvaro Uribe había decidido fusionar con los ministerios del Interior y de Protección Social. Con ello, temas cruciales de la política pública adquirieron mayor visibilidad. En segundo lugar, Santos decidió liquidar instituciones ineficientes, que se habían convertido en focos de corrupción. La Dirección Nacional de Estupefacientes (DNE), y el Departamento Administrativo de Seguridad (DAS) fueron los dos primeros entes que se abolieron. Algunas de las funciones que desempeñaban fueron asumidas por otras instituciones tales como el Ministerio del Interior y el de Relaciones Exteriores, la Fiscalía Nacional y la Policía Nacional. Al finalizar el año, quedó planteada la liquidación del Instituto Nacional Penitenciario y Carcelario (INPEC), e iniciado 2012 se empezó a dar la discusión al respecto (Ministerio del Interior y de Justicia, 2011).

En cuanto a políticas públicas, al menos tres leyes enigmáticas fueron sancionadas en 2011 por el presidente Santos. En junio el Congreso de la República aprobó en último debate la Ley de Víctimas, por la cual se dictan medidas de atención, asistencia y reparación integral a las víctimas del conflicto armado interno. El proyecto de ley había sido presentado por el gobierno días después de que Santos se posesionó como presidente. Su gestión a lo largo del proceso de discusión marcó una clara diferencia de posición frente a su antecesor, Uribe. Al no admitir este último la existencia de un conflicto armado interno (entre otras razones), la iniciativa que también fue presentada durante su gobierno no pudo ver la luz y fue archivada. La sanción de la ley significa un paso adelante en el reconocimiento de las víctimas, y en su reparación a través de la recuperación de las tierras que perdieron como consecuencia del conflicto. No obstante la dificultad por implementar tal política, la ley ha sido considerada tanto nacional como internacionalmente como un gran avance hacia la reconciliación (Montero Carvajal, Uribe, \& León, 2011).

En segundo lugar, también en junio, el Congreso aprobó la ley orgánica de ordenamiento territorial (LOOT), la cual dicta normas orgánicas para el ordenamiento del territorio colombiano, define el marco institucional e instrumentos para el desarrollo territorial, distribuye competencias entre la nación y las entidades territoriales y establece las normas generales para la organización territorial. Por último, lo que esta ley busca es lograr mayor desarrollo en el país, especialmente en las regiones más apartadas (Vanguardia, 2011). 
Finalmente, una ley que implicó una reforma constitucional y que también fue de iniciativa gubernamental, fue la de regalías aprobada a finales del año. Su propósito es lograr distribuir los recursos generados por la producción de petróleo, oro, carbón, gas y níquel entre los departamentos productores y aquellos no productores. Hasta el momento, solamente los departamentos productores recibían regalías, por lo cual aquéllos que no lo hacían podían quedar rezagados en su desarrollo. Aunque la discusión del proyecto suscitó gran oposición por parte de aquellos municipios que verían recortadas sus finanzas, la sanción de la ley llegó en diciembre. Según el gobierno, una esperada bonanza minera durante los próximos diez años significará la entrada de millonarios recursos. El argumento central para defender el proyecto se centró en que ante dicho contexto sería necesario garantizar el manejo eficiente de recursos. Para esto, se hacía necesaria la centralización del manejo de las regalías así como su redistribución a lo largo y ancho del país (El Espectador, 2011a; León, 2010).

\section{RESULTADOS ELECTORALES}

En 2011 se realizaron elecciones locales. A éstas las antecedieron las elecciones presidenciales y legislativas en 2010. En esta sección resumimos los resultados de unas y otras, y elaboramos un breve balance.

\subsection{Elecciones presidenciales y legislativas}

En marzo de 2010 se eligió un nuevo Congreso de la República conformado por 102 senadores y 163 representantes a la Cámara. Posteriormente, en mayo y junio se realizaron la primera y segunda vueltas electorales presidenciales, respectivamente. En la última, Juan Manuel Santos, del Partido Social de Unidad Nacional (Partido de la U), se impuso con el 69,1\% de los votos sobre su contendor Antanas Mockus, quien obtuvo el 27,5\% de la votación. Antes de presentar los resultados de las diferentes contiendas, es importante describir el contexto en el que éstas se realizaron. En primer lugar, vale la pena recordar que hasta febrero de 2010 se presentó una situación de incertidumbre sobre la posibilidad de que Álvaro Uribe se pudiera volver a presentar como candidato presidencial. En septiembre de 2009 el Presidente sancionó una ley que permitía su segunda reelección consecutiva. No obstante, en la revisión de constitucionalidad la Corte encontró vicios en el proceso de aprobación de la ley y en febrero del siguiente año la declaró inconstitucional. Solo fue en ese momento que Juan Manuel Santos definió su candidatura, la cual fue avalada por el Partido de la U y decididamente apoyada por el entonces mandatario (Congreso Visible, 2010). La influencia de Uribe sobre la agenda programática de la campaña de Santos fue clara. Éste último prometió dar continuidad a las políticas más enigmáticas del primero, fundamentalmente a la de "Seguridad Democrática".

En segundo lugar, la campaña presidencial la atravesó el que se conoció como el fenómeno de la "Ola Verde". Antes de la primera vuelta, el candidato del Partido Verde, Antanas Mockus, despertó en el electorado, especialmente entre los jóvenes, una gran acogida. Su discurso se enfocó en la lucha contra la corrupción y en la recuperación de los valores 
humanos. Por ejemplo, a lo largo de toda la campaña, sus seguidores se apropiaron de frases tales como "la vida es sagrada" o "a mí no me pagaron", entre otras. Fue una campaña original y muy emotiva, que presentó al candidato como alguien independiente y alejado de la politiquería y la corrupción. Paradójicamente, el motivo que parecía haberle dado mucha visibilidad y fuerza se convirtió en una de las explicaciones de su derrota: no contar con una maquinaria sólida que lo respaldara. Además, su partido apenas había logrado seis curules para el Congreso en las elecciones legislativas, lo cual también pudo haber incidido en la decisión final de algunos de quienes habían dicho que apoyaban a la ola. Al final, el fenómeno se desinfló, y el candidato obtuvo el $21,0 \%$ de los votos en la primera vuelta (3.120.716 votos) y no el $36 \%$ que las encuestas habían predicho. ${ }^{5}$

En tercer lugar, las elecciones de 2010 se presentaron como una nueva ocasión para que los ciudadanos contribuyeran a la renovación de la política a través de su participación. A lo largo del cuatrienio que había empezado en 2006, se presentó el que se conoció como el escándalo de la para-política. A través de éste se hizo público que más de 80 congresistas y varios funcionarios del gobierno tenían estrechos vínculos con grupos paramilitares (López, 2010). Uno de los resultados del escándalo fue que la gran mayoría de los legisladores involucrados perdieron la curul y varios recibieron condenas que los llevaron a la cárcel. Así, el 2010 se constituyó como un año en el que se ponía a prueba la legitimidad de las elecciones para el cuerpo representativo por excelencia.

Seis candidatos se constituyeron en los que definieron la campaña presidencial. Además de Santos y Mockus, se sumaron el ex senador "tradicional" del partido Cambio Radical, Germán Vargas Lleras, el representante del partido de izquierda Gustavo Petro (Polo Democrático); la otrora (1998) candidata presidencial Noemí Sanín por el Partido Conservador, y el entonces director del Partido Liberal, Rafael Pardo. Los Cuadros 2 y 3 resumen los resultados de las elecciones presidenciales.

Cuadro 2: Resultados elecciones presidenciales 2010, primera vuelta

\begin{tabular}{llcc}
\hline Candidato & Partido político & Votos & Porcentaje \\
\hline Juan Manuel Santos & Partido de la U & 6.758 .539 & 46,6 \\
Antanas Mockus & Partido Verde & 3.120 .716 & 21,5 \\
Germán Vargas Lleras & Cambio Radical & 1.471 .377 & 10,1 \\
Gustavo Petro & Polo Democrático & 1.329 .512 & 9,2 \\
Noemí Sanín & Conservador & 829.323 & 6,1 \\
Rafael Pardo & Liberal & 636.624 & 4,4 \\
\hline
\end{tabular}

Fuente: Registraduría Nacional del Estado Civil. Tomado de Hoskin y Pachón (2011).

5 Las últimas encuestas que se realizaron antes de la primera vuelta, predecían un empate técnico entre Juan Manuel Santos y Antanas Mockus: "Santos 38\% - Mockus 36\% en primera vuelta, según encuesta del CNC", en Revista Semana, 05/13/2010. 
Cuadro 3: Resultados elecciones presidenciales 2010, segunda vuelta

\begin{tabular}{llrc}
\hline \multicolumn{1}{c}{ Candidato } & Partido Político & \multicolumn{1}{c}{ Votos } & Porcentaje \\
\hline Juan Manuel Santos & Partido de la U & 9.004 .221 & 69,1 \\
Antanas Mockus & Partido Verde & 3.588 .819 & 27,5 \\
Votos no válidos y votos en blanco & & 744.618 & 3,4 \\
\hline Total & & 13.337 .658 & 100 \\
\hline
\end{tabular}

Fuente: Registraduría Nacional del Estado Civil. Tomado de Hoskin y Pachón (2011).

Cuadro 4: Resultado de las elecciones al Senado, 2006 y 2010

\begin{tabular}{|c|c|c|c|c|}
\hline \multirow{2}{*}{ Partido Político } & \multicolumn{2}{|c|}{2006} & \multicolumn{2}{|c|}{2010} \\
\hline & Votos & Escaños (\%) & Votos & Escaños (\%) \\
\hline Partido de la U* & 1.591 .775 & $20(19,6)$ & 2.549 .888 & $28(27,4)$ \\
\hline Partido Conservador* & 1.470 .029 & $18(17,6)$ & 2.257 .335 & $23(22,5)$ \\
\hline Partido Liberal ${ }^{*}$ & 1.436 .657 & $17(16,6)$ & 1.724 .151 & $17(16,6)$ \\
\hline Cambio Radical ${ }^{*}$ & 1.211 .457 & $15(14,7)$ & 861.816 & $8(7,8)$ \\
\hline Polo Democrático ${ }^{* *}$ & 875.451 & $11(10,7)$ & 824.948 & $8(7,8)$ \\
\hline $\mathrm{PIN}^{*}$ & 566.823 & $7(6,8)$ & 892.720 & $8(7,8)$ \\
\hline Alas Equipo Colombia & 418.124 & $5(4,9)$ & & NA \\
\hline Colombia Democrática & 272.524 & $3(2,9)$ & & NA \\
\hline $\operatorname{MIRA}^{* * *}$ & 237.512 & $2(1,9)$ & 324.109 & $2(1,9)$ \\
\hline Colombia Viva & 229.556 & $2(1,9)$ & & NA \\
\hline Partido Verde* & NA & NA & 521.503 & $5(4,9)$ \\
\hline $\begin{array}{l}\text { Alianza Social } \\
\text { Indígena*** }\end{array}$ & 43.903 & $1(0,9)$ & & $1(0,9)$ \\
\hline A.I.C.O ${ }^{* * *}$ & 21.624 & $1(0,9)$ & & $1(0,9)$ \\
\hline
\end{tabular}

Fuente: Registraduría Nacional del Estado Civil. Tomado de Hoskin y Pachón (2011).

(*) Partidos que integran la coalición legislativa "Unidad Nacional" convocada por el presidente Santos en 2010. Vale la pena aclarar que tanto el PIN como el Partido Verde se unieron recientemente a la coalición.

${ }^{* *}$ ) Partidos de oposición en $2010{ }^{* * *}$ ) Partidos independientes en 2010.

En la segunda vuelta electoral, Santos se constituyó en el presidente de los colombianos tras obtener la votación más alta en la historia política del país (Hoskin \& Pachón, 2011). En su discurso de posesión hizo un llamado a los congresistas elegidos en marzo para que lo acompañaran en su gestión. Fue así como a partir de julio, la mayoría de quienes integraron el cuerpo legislativo formaron parte de la gran coalición del gobierno, la 
llamada Unidad Nacional. Solamente el Polo Democrático y el Partido Verde se resistieron a integrarse en ella. El PIN por su parte no fue invitado a que lo hiciera por tener dentro de su bancada miembros cercanos a grupos ilegales. Los Cuadros 4 y 5 presentan los resultados de las elecciones legislativas en dos años: 2006 y 2010.

Los resultados de las elecciones legislativas de 2010 permitieron evaluar la evolución de la reforma electoral de 2003. Esta fue la tercera ocasión en la que se aplicaron las reglas

Cuadro 5: Resultado de las elecciones a la Cámara de Representantes, 2006 y 2010

\begin{tabular}{|c|c|c|c|c|}
\hline Partido Político & Votos & Escaños $(\%)$ & Votos & Escaños (\%) \\
\hline Partido de la $U^{*}$ & 1.453 .353 & $29(17,4)$ & 2.457 .916 & $48(28,9)$ \\
\hline Partido Conservador* & 1.363 .656 & $30(18,1)$ & 2.024 .326 & $36(21,6)$ \\
\hline Partido Liberal ${ }^{*}$ & 2.646 .404 & $36(21,6)$ & 1.756 .882 & $36(21,6)$ \\
\hline Cambio Radical* & 932.207 & $20(12)$ & 722.453 & $16(9,6)$ \\
\hline Polo Democrático A** & 708.664 & $9(5,4)$ & 585.101 & $5(3,1)$ \\
\hline PIN (Convergencia Ciudadana en 2006)* & 397.903 & $8(4,8)$ & 707.203 & $11(6,6)$ \\
\hline Alas (Equipo Colombia en 2006) & 370.789 & $7(4,2)$ & 183.534 & $1(0,6)$ \\
\hline Colombia Democrática & 215.753 & $2(1,2)$ & & NA \\
\hline MIRA*** $^{* *}$ & 233.920 & $1(0,6)$ & 324.505 & $1(0,6)$ \\
\hline Por el país que soñamos & 99.565 & $2(1,2)$ & & NA \\
\hline Movimiento Integración Regional ${ }^{*}$ & 91.547 & $4(2,4)$ & 5.093 & $1(0,6)$ \\
\hline Movimiento Popular Unido* & 129.977 & $2(1,2)$ & 31.764 & 0 \\
\hline Movimiento Participación Popular & s.i & $1(0,6)$ & & NA \\
\hline Movimiento Salvación Nacional & s.i & $1(0,6)$ & & NA \\
\hline Movimiento Nacional Progresista & s.i & $1(0,6)$ & & NA \\
\hline Huila Nuevo Liberalismo & 80.688 & $1(0,6)$ & & NA \\
\hline MORAL & s.i & $1(0,6)$ & & NA \\
\hline Partido Verde (Opción Centro en 2006)* & s.i & $1(0,6)$ & 292.228 & $3(1,8)$ \\
\hline Afrouninca ${ }^{* * *}$ & s.i & $1(0,6)$ & & NA \\
\hline Alianza Social Afrocolombiana*** & s.i & $1(0,6)$ & & NA \\
\hline Alianza Social Indígena ${ }^{* * *}$ & & NA & 206.460 & $1(0,6)$ \\
\hline Unidad Liberal* & & NA & 79.507 & $2(1,2)$ \\
\hline Apertura Libera*1 & 199.810 & $5(3,1)$ & 102.168 & $2(1,2)$ \\
\hline Total & & 163 & & \\
\hline
\end{tabular}

Fuente: Registraduría Nacional del Estado Civil. Tomado de Hoskin y Pachón (2011).

(*) Partidos que integran la coalición legislativa "Unidad Nacional" convocada por el presidente Santos en 2010. Vale la pena aclarar que tanto el PIN como el Partido Verde se unieron recientemente a la coalición.

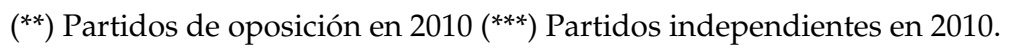


adoptadas en ese momento. En estos comicios se fortaleció la tendencia de disminución en el número de partidos que compitieron en la contienda, así como en el número efectivo de partidos (ver Gráfico 1) (Botero \& Rodríguez-Raga, 2006). El Partido de la U, el PL y el PC fueron las tres fuerzas que mayor poder lograron acumular. Más adelante, también con Cambio Radical se constituyeron en partes centrales de la coalición de gobierno.

En términos de volatilidad electoral, entendida como el cambio en las preferencias partidistas del electorado, no es significativo si se compara históricamente con las cifras de volatilidad en el país (en el caso de la Cámara) y si se compara también con los niveles de volatilidad de otros países de la región como Brasil $(24,1)$ o Argentina $(24,9)$. En Colombia, las últimas elecciones legislativas revelaron que en términos de la composición partidista, el cuerpo colegiado no varió tanto respecto a 2006. El Cuadro 6 ilustra esta idea.

Finalmente, la depuración del Congreso todavía está por verse. El día de la elección se hizo claro que los actores ilegítimos siguen permeando a la institución, y que nuevamente pudieron volver a poner sus fichas. Uno de los partidos que ha sido fuertemente criticado por ello es el Partido de Integración Nacional (PIN), el cual sumó en 2010 aquellos partidos que en 2006 habían elegido a quienes fueron destituidos por la para-política: Colombia Viva, Colombia Democrática y Convergencia Ciudadana.

Gráfico 1: Número efectivo de partidos, 2006-2010
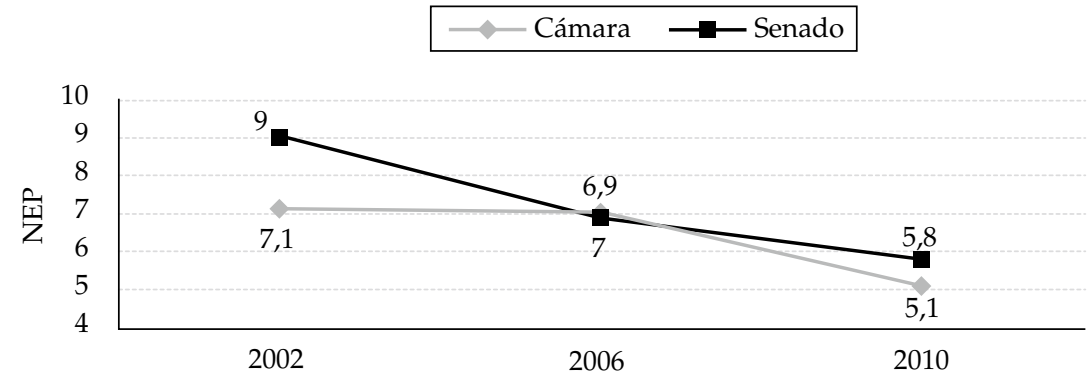

Cuadro 6: Volatilidad electoral medida a partir de los escaños ${ }^{6}$

\begin{tabular}{lcc}
\hline & $1978-2002^{7}$ & $2006-2010$ \\
\hline Cámara & 22,1 & 20,1 \\
Senado & N.D & 19,5 \\
\hline
\end{tabular}

Cálculos propios a partir de resultados electorales. 


\subsection{Elecciones locales}

El 30 de octubre se eligieron 32 gobernaciones, 418 puestos a Asambleas Departamentales, 1.102 alcaldías municipales, 12.612 concejales y 4.958 ediles. Más de 100.000 candidatos, avalados por 12 partidos políticos y varios movimientos ciudadanos compitieron por estos cargos. La participación ciudadana fue en promedio cercana al $60 \%$, no obstante varió según los cargos a elegir y a través del territorio nacional. ${ }^{8}$ En estos comicios se aplicaron por tercera vez, en elecciones locales, las reglas electorales definidas en la reforma política de 2003. ${ }^{9}$ Adicionalmente, nuevas disposiciones aprobadas en 2009 y reglamentadas a mediados de 2011 empezaron a regir en esta jornada electoral. ${ }^{10}$

Entre las medidas adoptadas, se incluyó una "ley de cuotas" que definió que todas las listas partidistas tenían que incluir al menos 30\% de mujeres. Esta decisión se tomó cuando ya muchos partidos habían configurado sus listas. Fue necesario entonces la redefinición de las mismas muy poco tiempo antes del día de las elecciones. El balance que varios líderes hicieron sobre la medida fue mixto. Si bien consideraban que la cuota podía ayudar a incrementar la participación política de las mujeres, su aplicación prematura condujo a varios partidos a incluir lo que llamaron "mujeres de relleno". En cuanto a los resultados, no hubo un incremento real en la porción de poder político ocupado por mujeres. Por otro lado, la reforma estableció nuevas exigencias a los partidos relacionadas con la necesidad de depurar sus listas para con ello disminuir el riesgo de incluir candidatos vinculados con la criminalidad. Como resultado de esto, los diferentes partidos consultaron con el Ministerio del Interior la situación de 167.828 aspirantes a candidaturas, el que encontró 13.833 hallazgos de antecedentes penales, fiscales y/o disciplinarios. A pesar de este esfuerzo, los primeros balances de los resultados mostraron que varios candidatos elegidos fueron apoyados por actores ilegítimos (Verdad Abierta, 2011). Un cambio adicional fue la facilitación de las condiciones para la formación de movimientos ciudadanos. Como lo muestra el mapa 1, varios de los nuevos actores que surgieron como resultado de ello lograron acceder al poder. Adicionalmente, se estableció la posibilidad de constituir alianzas interpartidistas para la competencia a los cargos uninominales (i.e. alcaldías y gobernaciones). Muchos candidatos lograron el apoyo de varios partidos. Un ejemplo que ilustra esta situación lo constituyó la candidatura a la gobernación de Cundinamarca por parte de Álvaro Cruz, quien recibió el apoyo de cuatro organizaciones: los dos partidos tradicionales -el PL y el PC-; Cambio Radical; y el Partido de la U. Una pregunta interesante que surge de esta situación tiene que ver con la identidad partidista de los mandatarios elegidos, así como con la definición de las agendas programáticas y con la responsabilidad política hacia el electorado.

El Cuadro 7 resume información general sobre las elecciones. Los mapas 1 y 2 ilustran de manera comparada con los resultados de 2007, las gobernaciones y las alcaldías de las capitales departamentales ganadas por los diferentes partidos políticos. Salta a la

8 En Colombia el voto no es obligatorio.

9 La reforma de 2003 estuvo dirigida a organizar el sistema de partidos a través de la generación de incentivos para cohesionar a los partidos políticos.

10 Ley 1465 de 2011, sancionada el 14 de julio. 
vista que el colorido de los mapas cambió casi que en su totalidad de una elección a la siguiente, y que en las elecciones de 2011 gran cantidad de movimientos ciudadanos lograron elegir a sus candidatos.

Cuadro 7: Elecciones locales, 2011. Información general.

\begin{tabular}{|c|c|c|c|c|c|c|c|}
\hline Corporación & $\begin{array}{l}\text { Cargos } \\
\text { a elegir }\end{array}$ & $\begin{array}{l}\text { Potencial } \\
\text { Sufragantes }\end{array}$ & $\begin{array}{l}\text { Sufragantes } \\
(\%)\end{array}$ & $\begin{array}{c}\text { Votos } \\
\text { blancos } \\
(\%)\end{array}$ & $\begin{array}{c}\text { Votos } \\
\text { válidos } \\
(\%)\end{array}$ & $\begin{array}{c}\text { Votos } \\
\text { Nulos } \\
(\%)\end{array}$ & $\begin{array}{c}\text { Votos no } \\
\text { marcados } \\
(\%)\end{array}$ \\
\hline JAL & 4958 & 14.724.117 & $\begin{array}{c}7.678 .218 \\
(51,1)\end{array}$ & $\begin{array}{c}1.708 .368 \\
(22,2)\end{array}$ & $\begin{array}{c}6.380 .072 \\
(83,1)\end{array}$ & $\begin{array}{c}360.602 \\
(4,7)\end{array}$ & $\begin{array}{c}937.544 \\
(12,2)\end{array}$ \\
\hline Concejos & 12612 & 30.615 .748 & $\begin{array}{c}17.186 .217 \\
\quad(56,1)\end{array}$ & $\begin{array}{c}922.201 \\
(5,)\end{array}$ & $\begin{array}{c}15.628 .124 \\
(90,9)\end{array}$ & $\begin{array}{c}846.209 \\
(4,9)\end{array}$ & $\begin{array}{c}711.884 \\
(4,1)\end{array}$ \\
\hline Alcaldías & 1102 & 30.615 .748 & $\begin{array}{c}17.480 .981 \\
(57,1)\end{array}$ & $\begin{array}{c}646.688 \\
(3,7)\end{array}$ & $\begin{array}{c}16.565 .616 \\
(94.8)\end{array}$ & $\begin{array}{c}388.606 \\
(2.2)\end{array}$ & $\begin{array}{c}526.759 \\
(3)\end{array}$ \\
\hline Asambleas & 418 & 25.764 .448 & $\begin{array}{c}14.820 .216 \\
(57,5)\end{array}$ & $\begin{array}{c}1.533 .721 \\
(10,3)\end{array}$ & $\begin{array}{c}12.416 .217 \\
(83,8)\end{array}$ & $\begin{array}{c}693.252 \\
(4,7)\end{array}$ & $\begin{array}{c}1.710 .747 \\
(11,5)\end{array}$ \\
\hline Gobernaciones & 32 & 25.764 .448 & $\begin{array}{c}15.061 .378 \\
(58,5)\end{array}$ & $\begin{array}{c}1.052 .179 \\
(7)\end{array}$ & $\begin{array}{c}13.144 .654 \\
(87,3)\end{array}$ & $\begin{array}{c}337.367 \\
(2,2)\end{array}$ & $\begin{array}{c}1.579 .357 \\
(10,5)\end{array}$ \\
\hline
\end{tabular}

Fuente: Registraduría Nacional del Estado Civil. Elaboración propia.

Mapa 1: Elecciones locales: gobernaciones por partido político
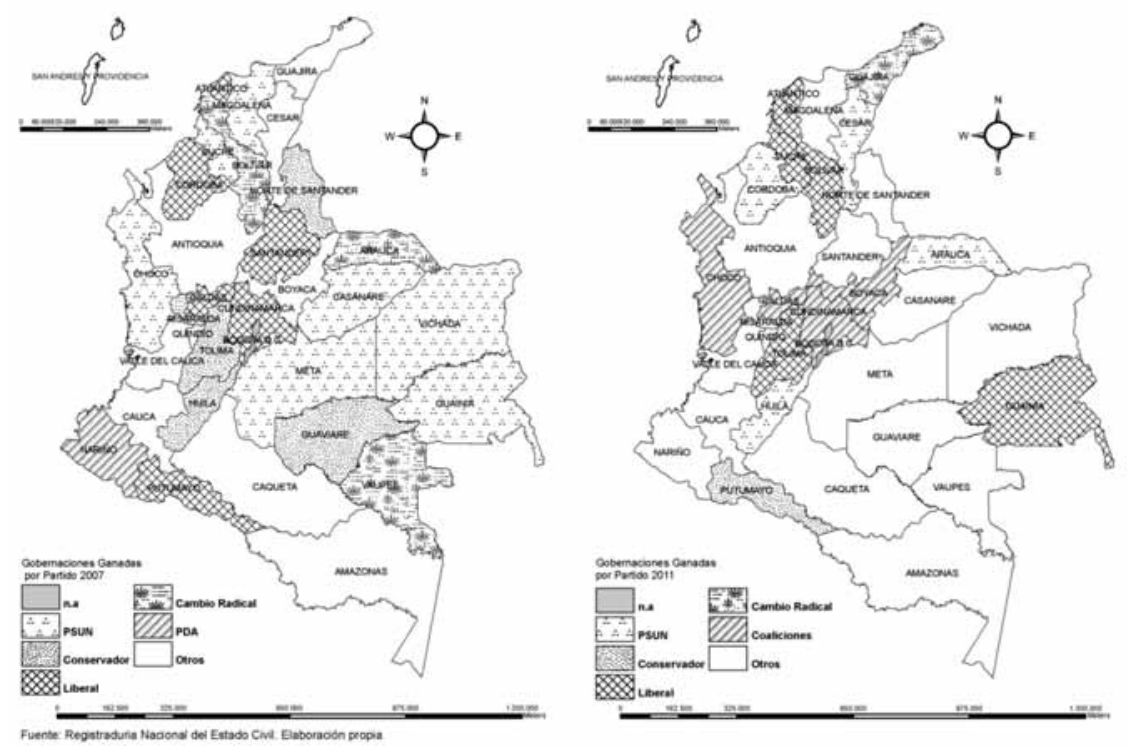
Mapa 2: Elecciones locales: alcaldías capitales de departamento por partido político
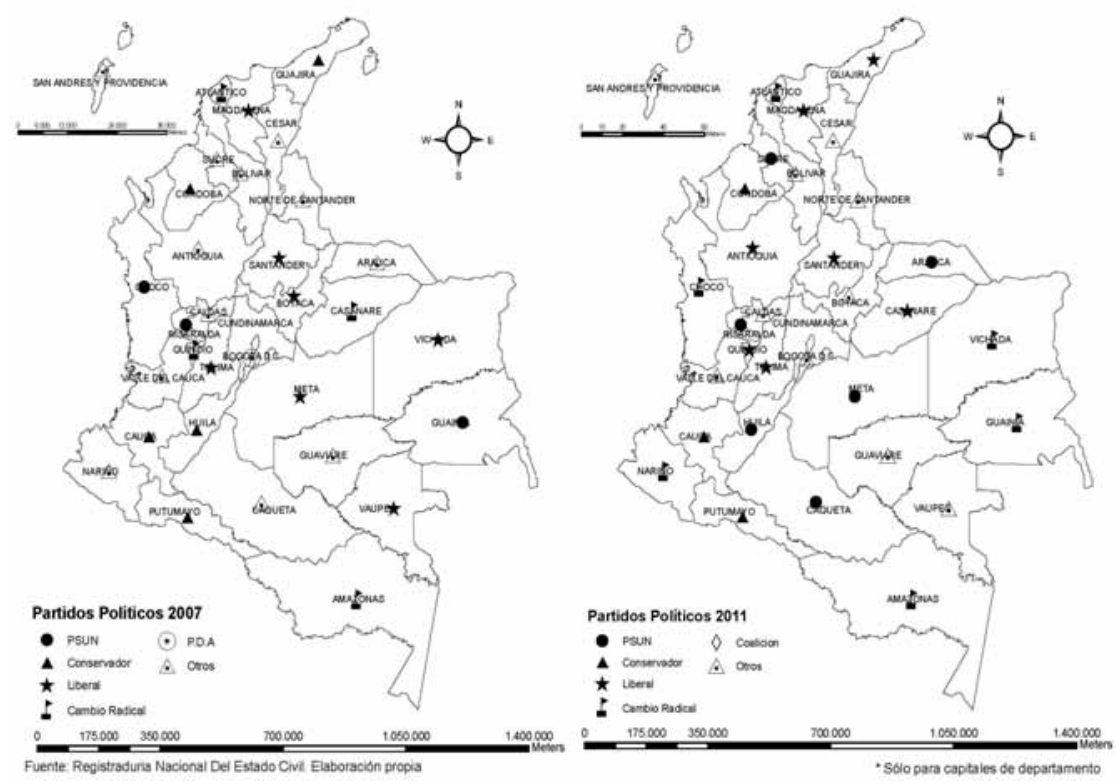

En conclusión, las elecciones locales de 2011 se sumaron a las nacionales de 2010. Las contiendas locales se desarrollaron bajo un nuevo marco legal. Aunque fue posible vislumbrar algunos cambios, tendrán que pasar al menos otras elecciones para poder realizar un análisis de impacto de las mismas. Las elecciones de 2010 se constituyeron en aquellas en las que después de ocho años de gobierno se eligió un nuevo presidente. En el Congreso se pudieron ver con mayor precisión los efectos de la reforma electoral de 2003: el número de partidos siguió disminuyendo. Sin embargo, la competencia al interior de las listas intrapartidarias ha seguido dándose. Las elecciones locales mostraron que los partidos de la coalición del gobierno (PSUN, PL, PC, Cambio Radical) obtuvieron una alta porción del poder electoral. No obstante, es importante subrayar los resultados de Bogotá donde Gustavo Petro del movimiento de izquierda "Progresistas" triunfó. Por tercera vez consecutiva ganó en la capital del país el candidato de tendencia ideológica opuesta a la del Presidente de la República. Pareciera ser que las preferencias electorales de los bogotanos se diferencian de las de la mayoría del país. Dados el alto porcentaje de personas que votan en la capital y la importancia del cargo -la alcaldía de Bogotá se conoce como el segundo cargo de elección popular más importante del país-, este hecho se constituye como uno de gran significado. En las siguientes secciones veremos la configuración del gabinete ministerial y el estilo de gobierno, así como el desempeño legislativo del Congreso durante el 2011 y las relaciones entre las dos ramas. 


\section{PODER EJECUTIVO: EL GABINETE MINISTERIAL Y EL ESTILO DE GOBIERNO}

Tras la posesión de Juan Manuel Santos como Presidente de Colombia, una de las decisiones más esperadas por el país fue la relacionada con la conformación de su gobierno, específicamente la selección de los ministros. Se puede decir que el gabinete del actual gobierno es una mezcla entre tecnócratas y políticos de profesión, lo cual denota una diferencia con respecto a los gabinetes de su predecesor Álvaro Uribe. La prueba fehaciente de que Santos consiguió un equilibrio entre un componente técnico y un componente político en su gabinete es la información sobre el perfil de los actuales ministros. Por ejemplo, carteras como la de Hacienda y Crédito Público en cabeza de Juan Carlos Echeverry, el Ministerio de Defensa a cargo de Juan Carlos Pinzón o el Ministerio de Educación dirigido por María Fernanda Campo, demuestran como temas asociados a una agenda política tradicional que se encuentran en manos de técnicos especializados en la materia cuya participación en el sector público ha sido nula o muy poca y por el contrario su experiencia en empresas del sector privado o la academia resulta significativa.

No obstante, las decisiones tomadas por Santos para la conformación de su gabinete dejan ver que la participación de políticos de profesión es también fundamental para el gobierno. La presencia de Germán Vargas Lleras, Rafael Pardo y Juan Camilo Restrepo -los primeros dos ex candidatos presidenciales y competencia de Santos en 2010- en las carteras del Interior, del Trabajo y de Agricultura respectivamente demuestran no sólo la presencia de figuras con experiencia en cargos de elección popular en el gobierno, sino además la intención de Santos de que su gabinete cuente con una representación partidista proporcional que armonice y se articule con la gran coalición legislativa que conformó días después de ser elegido como primer mandatario del país. La lista 1 enumera las diferentes carteras y describe información básica de sus representantes.

\section{Lista 1: Gabinete Ministerial de la Presidencia de Juan Manuel Santos, 2010-2011}

1. Ministerio de Agricultura y Desarrollo Rural: Juan Camilo Restrepo (1946 masculino, Partido Conservador). Inició con el gobierno. Es abogado y economista y ha ocupado cargos en el sector público, incluyendo ministerios.

2. Ministerio de Comercio, Industria y Turismo: Sergio Diazgranados (1968 masculino, Partido de la U). Inició con el gobierno. Es abogado, y ha ocupado cargos de elección popular.

3. Ministerio de Minas y Energía: Mauricio Cárdenas (1962 masculino, Partido Conservador). Fue nombrado en septiembre de 2011 en reemplazo de Carlos Rodado Noriega. Es economista, y ha ocupado cargos en el sector público, incluyendo ministerios.

4. Ministerio de Hacienda y Crédito Público: Juan Carlos Echeverry (1962, masculino, Partido Conservador). Inició con el gobierdno. Es economista, y ha ocupado cargos 
directivos en el sector público. También se ha desempeñado en la academia y en el sector privado.

5. Ministerio de Transporte: Germán Cardona (1956, masculino, Partido de la U). Inició con el gobierno. Es ingeniero civil, y ha ocupado cargos de elección popular.

6. Ministerio de Relaciones Exteriores: María Ángela Holguín (s.i, femenino, Independiente). Inició con el gobierno. Es politóloga, y ha ocupado cargos en el sector público así como en el sector privado.

7. Ministerio del Interior: Germán Vargas Lleras (1962, masculino, Cambio Radical). Inició con el gobierno. Es abogado, y ha ocupado cargos de elección popular. Fue candidato presidencial en 2010.

8. Ministerio de Defensa: Juan Carlos Pinzón (1972, masculino, tendencia conservadora). Fue nombrado en agosto de 2011 en reemplazo de Rodrigo Rivera Salazar. Es economista, y ha ocupado cargos en el sector público y en el sector privado.

9. Ministerio de Educación: María Fernanda Campo (s.i, femenino, Independiente). Inició con el gobierno. Es ingeniera industrial y ha ocupado cargos en el sector público y en el sector privado.

10. Ministerio de Cultura: Mariana Garcés (s.i., femenino, Independiente). Inició con el gobierno. Es abogada y ha ocupada cargos en el sector público.

11. Ministerio de Ambiente, Vivienda y Desarrollo Territorial: Beatriz Uribe Botero (s.i., femenino, Independiente). Inició con el gobierno. Es economista y ha ocupado cargos en el sector público.

12. Ministerio de Tecnologías de la Información y Comunicaciones: Diego Molano Vega (1967, masculino, Partido Liberal). Inició con el gobierno. Es ingeniero electrónico y ha ocupado cargos en el sector público así como en el sector privado.

13. Ministerio de Trabajo: Rafael Pardo Rueda (1953, masculino, Partido Liberal). Fue nombrado en octubre de 2011 tras la separación del ministerio de la cartera de Salud y Protección Social. Es economista y ha ocupado cargos de elección popular. Fue candidato presidencial en 2010.

14. Ministerio de Salud: Beatriz Londoño Soto (s.i., femenino, Independiente). Fue nombrada en enero de 2012, después de que el Ministerio de Trabajo se separara del anterior Ministerio de Salud y Protección Social. Reemplazó a Mauricio Santamaría. Es médica y ha ocupado cargos en el sector privado y también en el público.

15. Ministerio de Justicia: Juan Carlos Esquerra (1949, masculino, cercano al Partido Liberal). Es abogado y ha ocupado cargos en los sectores público y privado. Fue nombrado en julio 2011 tras la separación del ministerio de la cartera del Interior.

16. Ministerio de Medio Ambiente: Frank Pearl (s.i, masculino, Independiente). Es economista y ha ocupado cargos en el sector público y en el privado. Fue nombrado en 2011. 
Cuadro 8: Primer año de actividad legislativa: julio 2010 - marzo 2011

\begin{tabular}{lcccc}
\hline \multicolumn{1}{c}{ Iniciativa } & $\begin{array}{c}\text { Radicados } \\
(\%)\end{array}$ & $\begin{array}{c}\text { Aprobados en } \\
\text { últimos debates }\end{array}$ & $\begin{array}{c}\text { \% tasa de aprobación } \\
\text { sobre el total }\end{array}$ & $\begin{array}{c}\text { Permanecen } \\
\text { en debates }\end{array}$ \\
\hline Legislativa & $365(84)$ & 9 & 2 & 126 \\
Gubernamental & $65(15)$ & 23 & 35 & 24 \\
Otras Entidades & $2(0,5)$ & 2 & 100 & 0 \\
\hline Total & 432 & 34 & & \\
\hline
\end{tabular}

Fuente: Congreso Visible, 2011.

Cuadro 9: Número de proyectos por tipo de iniciativa (julio - diciembre de 2011)

\begin{tabular}{lc}
\hline \multicolumn{1}{c}{ Iniciativa } & $\mathrm{N}^{\circ}$ de proyectos $(\%)$ \\
\hline Legislativa & $238(81)$ \\
Gubernamental & $53(18)$ \\
Otras entidades & $2(1)$ \\
\hline Total & 293 \\
\hline
\end{tabular}

Fuente: Congreso Visible, 2011.

Gráfico 2: Temas de los proyectos discutidos en el primer año de actividad legislativa $\mathrm{N}^{\mathrm{o}}$ de Proyectos

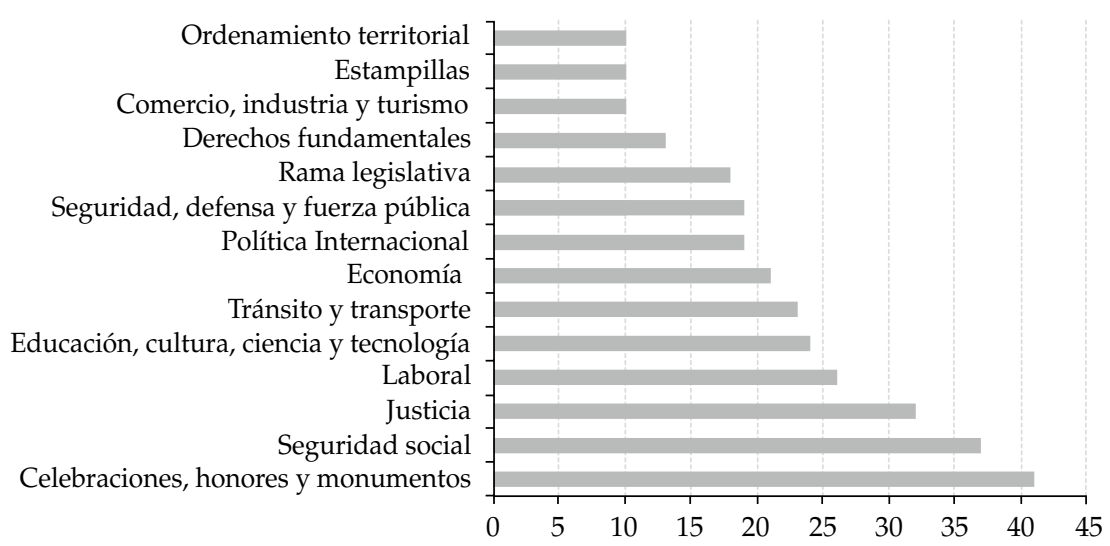

Fuente: Congreso Visible, 2011. 


\section{PODER LEGISLATIVO}

A pesar de que el primer año y medio de actividad legislativa en Colombia estuvo atravesado por la coyuntura electoral se observa, por un lado, un alto nivel de producción legislativa durante este periodo y, por otro, un papel bastante activo por parte del Ejecutivo en la elaboración y presentación de proyectos de ley. Un análisis general del desempeño por parte del Congreso en este periodo deja un balance positivo para el Ejecutivo: en términos proporcionales el gobierno registró una tasa de aprobación de más del 30\% de los proyectos que presentó ante el Legislativo. Tal nivel de éxito es un indicativo sobre la alta coordinación que se ha dado entre los partidos que conforman la coalición del gobierno, la Unidad Nacional, y los ministros del gabinete.

Si bien la mayoría de las iniciativas que han sido presentadas y discutidas en el Congreso se asocian a celebraciones, honores y monumentos, es importante destacar el protagonismo que temas como la seguridad social, la justicia y las políticas laborales han tenido en la agenda legislativa en los primeros dieciocho meses de actividad legislativa. Dentro de los proyectos más importantes que han sido discutidos y sancionados como leyes de la república, se destacan la Ley de Víctimas, la Ley de Ordenamiento Territorial, la reestructuración de la rama administrativa, y la Ley de Regalías. Más recientemente, entró en discusión un proyecto de ley también de iniciativa gubernamental, que busca llevar a cabo una reforma al sistema judicial del país. Se espera asimismo la presentación de un nuevo proyecto de reforma tributaria (Congreso Visible, 2012). Los Cuadros 8 y 9 y el Gráfico 2 resumen la actividad legislativa desde julio de 2010 hasta diciembre de 2011.

En materia de control político, vale la pena destacar el rol activo que los partidos de la coalición, especialmente el Partido de la U -partido del Presidente-, y los partidos tradicionales han tenido a la hora de llamar a debates de control político a los ministros. Por su parte, el único partido de oposición, el Polo Democrático Alternativo, ha hecho un esfuerzo importante por adelantar citaciones a ministros. En veitiún oportunidades ha llamado a los representantes del gobierno para que rindan cuentas frente al Legislativo. Los ministros de Hacienda y Crédito Público, Transporte y Defensa son los que han sido citados con más frecuencia.

\section{CONCLUSIONES}

\section{Evaluación general sobre el funcionamiento y la calidad de la democracia}

En este artículo se reseñaron diferentes procesos que permearon la política nacional a lo largo de 2011. Como es tradición en Colombia, los procesos electorales que legitiman el sistema democrático se entremezclaron con la continuación del conflicto armado interno que lleva desangrando al país más de cinco décadas. Acá se mostró que la llegada al poder de un nuevo gobierno marcó diferencias en estilos presidenciales, así como en decisiones de políticas públicas. Aunque el gobierno de Juan Manuel Santos ha revelado ser más conciliador y menos autoritario que el de Uribe (Rodríguez-Raga \& Seligson, 2011), siguen por delante grandes desafíos que pondrán a prueba la buena voluntad del mandatario. 


\section{REFERENCIAS}

Arias Ortiz, A. (2012). Las "Bacrim" retan a Santos. Observatorio del Conflicto. Retrieved from http:/ /www. nuevoarcoiris.org.co/sac/?q=node/1394

Banco de la República. (2010). Regla Fiscal para Colombia. In M. d. H. y. C. Público (Ed.).

Botero, F., \& Rodríguez-Raga, J. C. (2006). Ordenando el caos: elecciones legislativas y reforma electoral en Colombia. Revista de Ciencia Política, 26(1), 138-151.

Congreso Visible. (2010). Congreso Visible. Balance del cuatrienio 2006-2010. Bogotá: Universidad de los Andes. Congreso Visible. (2012). Boletín No. 23. Bogotá: Universidad de los Andes.

CVNE. (2011). Cámara de Representantes oficializó retiro de reforma a la educación superior. Retrieved from http://www.mineducacion.gov.co/cvn/1665/w3-article-289661.html.

ElEspectador. (2011a,12/16/2011). Congreso aprueba proyecto deley deregalías. El Espectador. Retrieved from http:/ / www.elespectador.com/noticias/politica/articulo-316978-congreso-aprueba-proyecto-de-ley-de-regalias

El Espectador. (2011b,10/21/2011). Obama firmó el TLC Colombia-Estados Unidos. El Espectador. Retrieved from http://www.elespectador.com/economia/articulo-306720-obama-firmo-el-tlc-colombia-estados-unidos

El Mundo. (2010, 07/31/2010). El líder de las FARC propone diálogo al nuevo gobierno de Santos. El Mundo. Retrieved from http://www.elmundo.es/america/2010/07/30/colombia/1280513835.html

El Nuevo Herald. (2011, 11/25/2011). Críticas de Uribe a reunión Santos-Chávez encienden polémica en Colombia. El Nuevo Herald. Retrieved from http:/ / www.elnuevoherald.com/2011/11/25/1072403/ criticas-de-uribe-a-reunion-santos.html\#storylink=cpy

El Nuevo Siglo. (2011, 10/23/2011). Alerta por oleada de ataques de las Farc. El Nuevo Siglo. Retrieved from http://www.elnuevosiglo.com.co/articulos/10-2011-alerta-por-oleada-de-ataques-de-las-farc.html

El País. (2010, 08/10/2010). Colombia y Venezuela sellan la reconciliación tras cinco años de crisis. El País. Retrieved from http://internacional.elpais.com/internacional/2010/08/10/ actualidad/1281391208_850215.html

El País. (2011, 11/16/2011). Universitarios suspenden paro y piden que el Gobierno cumpla las condiciones. El País. Retrieved from http://www.elpais.com.co/elpais/colombia/noticias/ reforma-ley-30-quedo-hundida-definitivamente

El Tiempo. (2011a, 03/31/2011). A partir de mañana, Colombia preside Consejo de Seguridad de la ONU. El Tiempo. Retrieved from http://www.eltiempo.com/archivo/documento/CMS-9099680

El Tiempo. (2011b, 11/20/2011). 'Timochenko' le envía un fuerte comunicado al presidente Santos. El Tiempo. Retrieved from http://www.eltiempo.com/colombia/otraszonas/ARTICULO-WEBNEW_NOTA_INTERIOR-10799385.html

El Tiempo. (2011c, 06/07/2011). Ya son 27 los ataques de las Farc en Cauca durante este año. El Tiempo. Retrieved from http://www.eltiempo.com/colombia/occidente/ARTICULO-WEB-NEW_NOTA_ INTERIOR-9561944.html

Hoskin, G., \& Pachón, M. (2011). Colombia 2010: An Analysis of the Legislative and Presidential Elections. Colombia Internacional, 74, 9-26.

La Silla Vacía. (2011, 04/07/2011). Los cinco puntos que enfrentan a los estudiantes y profesores con la Ministra. La Silla Vacía. Retrieved from http://www.lasillavacia.com/historia/ los-cinco-puntos-que-enfrentan-los-estudiantes-y-profesores-con-la-ministra-23117

Lares, V. (2011,03/04/2011). Trece acuerdos comerciales entre Colombia y Venezuela. El Tiempo. Retrieved from http:/ / www.eltiempo.com/archivo/documento/MAM-4428442

León, J. (2010). Las cinco piezas que Santos mueve con la Ley de Regalías. Retrieved from http:/ / www. lasillavacia.com/historia/17711

León, J. (2011, 02/18/2011). Los camioneros le midieron el aceite al gobierno Santos. La Silla Vacía. Retrieved from http://www.lasillavacia.com/historia/los-camioneros-le-midieron-el-aceite-juan-manuel-santos-21780

León, J. (2012, 01/10/2012). Glosas a la carta de Timochenko. La Silla Vacía. Retrieved from http:/ / www. lasillavacia.com/historia/glosas-la-carta-de-timochenko-30619

López, C. (Ed.). (2010). Y refundaron la patria... De cómo mafiosos y políticos reconfiguraron el Estado colombiano. Bogotá: Corporación Nuevo Arco Iris and Random House Mondadori S.A. 
Ministerio del Interior y de Justicia. (2011). Reforma al Estado, más que tres nuevos ministerios. Retrieved from http:/ / www.dne.gov.co/?idcategoria=13359.

Montero Carvajal, D., Uribe, P. M., \& León, J. (2011, 06/1/2011). ¡Aprobada la ley de víctimas! La Silla Vacía. Retrieved from http:/ /www.lasillavacia.com/historia/aprobada-la-ley-de-victimas-24894

Portafolio. (2011a, 01/21/2011). 2 de febrero paro de camioneros por la eliminación de la tabla de fletes. Portafolio. Retrieved from http://www.portafolio.co/economia/2-febrero-paro-camioneros

Portafolio. (2011b, 11/04/2011). Colombia y Ecuador fortalecen la integración. Portafolio.

Revista Dinero. (2011, 02/01/2011). Ministro de Hacienda preocupado por alto desempleo. Revista Dinero.

Rodríguez-Raga, J. C., \& Seligson, M. A. (2011). Cultura política de la democracia en Colombia, 2011. Actitudes democráticas en la sucesión. Bogotá: Latin American Public Opinion Project.

Semana. (2010,08/10/2010). Santos y Chávez decidieron "anteponer las necesidades de nuestros pueblos". Semana.

Semana. (2011a, 04/22/2011). Centro del país: el más afectado por la ola invernal. Semana.

Semana. (2011b, 11/26/2011). Farc asesina a cuatro miembros de la fuerza pública secuestrados. Semana.

Semana. (2011c, 05/09/2011). María Emma Mejía por la integración suramericana. Semana.

Semana. (2011d, 02/17/2011). Según preacuerdo, Gobierno retira decretos que motivaron paro camionero. Semana.

Tickner, A. (2011, 12/20/2011). Colombia y el mundo 2011. El Espectador. Retrieved from http://www. elespectador.com/impreso/opinion/columna-317798-colombia-elmundo-2011

Vanguardia. (2011, 06/14/2011). La Ley orgánica de ordenamiento territorial pasó sanción presidencial. Vanguardia. Retrieved from http://www.vanguardia.com/ historico/108736- la-ley-organica-de-ordenamiento-territorial-paso-sancion-presidencial

Vargas, M. (2011, 05/21/2011). Los costos del invierno ya superaron los $\$ 4,6$ billones. Retrieved from http://www.eluniversal.com.co/monteria-y-sincelejo/economica/ los-costos-del-invierno-ya-superaron-los-46-billones-25303

Verdad Abierta. (2011). ¿Una nueva generación de parapolíticos? Paramilitarismo y conflicto armado en Colombia. Retrieved from http://www.verdadabierta.com/component/content/ article/63-nacional/3633-iuna-nueva-generacion-de-parapoliticos

Laura Wills-Otero. PhD en Ciencia Política de la Universidad de Pittsburgh (2010). Actualmente es profesora asistente en el Departamento de Ciencia Política de la Universidad de los Andes, Bogotá, Colombia. Coeditora de Colombia Internacional, revista de Ciencia Política de los Andes. Sus publicaciones más recientes han aparecido en capítulos de libros y en las revistas Controversia, Latin American Politics and Society; Journal of Politics in Latin America, the Journal of Women, Politics and Policy, Revista Colección.

E-mail: 1.wills21@uniandes.edu.co

Christian Iván Benito. Politólogo de la Pontificia Universidad Javeriana de Bogotá, Colombia y acualmente es estudiante de Maestría de Ciencia Política en la Universidad de los Andes. Trabajó en el programa Congreso Visible, y actualmente es asistente de investigación.

E-mail: ci.benito20@uniandes.edu.co 
